\title{
Tauroursodeoxycholic acid suppresses endoplasmic reticulum stress in the chondrocytes of patients with osteoarthritis
}

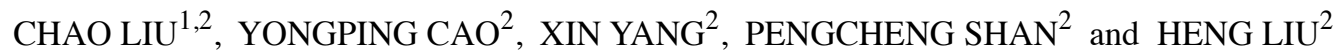 \\ ${ }^{1}$ Department of Orthopedics, Xinqiao Hospital, Third Military Medical University, Chongqing 400037; \\ ${ }^{2}$ Department of Orthopedics, Peking University First Hospital, Beijing 100034, P.R. China
}

Received November 24, 2014; Accepted June 26, 2015

DOI: 10.3892/ijmm.2015.2295

\begin{abstract}
The main pathogenic events in osteoarthritis (OA) include loss and abnormal remodeling of cartilage extracellular matrix. The present study aimed to evaluate the protective effect of tauroursodeoxycholic acid on chondrocyte apoptosis induced by endoplasmic reticulum (ER) stress. Articular cartilage tissues were collected from 18 patients who underwent total knee arthroplasty and were analyzed histologically. Subsequently, chondrocyte apoptosis was assessed by TUNEL. Quantitative polymerase chain reaction and western blot analysis were employed to evaluate gene and protein expression, respectively, of ER stress markers, including glucose-regulated protein 78 (GRP78), growth arrest and DNA-damage-inducible gene 153 (GADD153) and caspase-12 along with type II collagen. Chondrocytes obtained from osteoarthritis patients at different stages were cultured in three conditions including: No treatment (CON group), tunicamycin treatment to induce ER stress (ERS group) and tauroursodeoxycholic acid treatment after $4 \mathrm{~h}$ of tunicamycin (TDA group); and cell proliferation, apoptosis, function and ER stress level were assessed. Degradation of cartilage resulted in histological damage with more apoptotic cartilage cells observed. Of note, GRP78, GADD153 and caspase-12 mRNA and protein expression increased gradually from grade I to III cartilage tissue, while type II collagen expression decreased. Tunicamycin induced ER stress, as shown by a high expression of ER stress markers, reduced cell proliferation, increased apoptosis and decreased synthesis of type II collagen. Notably, tauroursodeoxycholic acid treatment resulted in the improvement of tunicamycin-induced ER stress. These results indicated that ER stress is highly involved in the tunicamycin-induced apoptosis in chondrocytes, which can be prevented by tauroursodeoxycholic acid.
\end{abstract}

Correspondence to: Dr Yongping Cao, Department of Orthopedics, Peking University First Hospital, Beijing 100034, P.R. China E-mail: caoyongpingmedsci@126.com

Key words: chondrocyte, osteoarthritis, endoplasmic reticulum stress, tauroursodeoxycholic acid, tunicamycin

\section{Introduction}

Osteoarthritis (OA) is an aging-associated disease (1) that affects the hyaline articular cartilage and all weight-bearing joint structures. The main pathogenic events in OA include loss and abnormal remodeling of cartilage extracellular matrix (2). Chondrocytes, the only cell type of the articular cartilage, maintain tissue homeostasis, respond to injury and perform the cartilage remodeling process that characterizes OA. Apoptosis has been proposed as a possible pathway for osteoarthritis pathology and a strong association has been demonstrated between the programmed cell death in chondrocytes and cartilage degradation in human osteoarthritis $(3,4)$. In addition, recent findings suggest that chondrocyte apoptosis is closely associated with cartilage matrix integrity and development of OA $(5,6)$.

Apoptosis in chondrocytes results mainly from mitochondrial and extrinsic pathways $(7,8)$. However, Archer and Francis-West (9) suggested that blocking these two pathways cannot completely prevent chondrocyte apoptosis and degradation of cartilage matrix, indicating that another pathway is responsible for apoptosis in chondrocytes. Recently, the endoplasmic reticulum (ER) pathway has been reported to be associated with apoptosis of chondrocytes in animal models and cultured cells. Yang et al (10) have reported that ER stress induces rat chondrocyte apoptosis and decreases the mRNA expression of extracellular matrix proteins, including aggrecan and type II collagen in articular cartilage. In addition, ER stress has been reported in chondrocytes in human osteoarthritis cartilage $(11,12)$. A recent study suggested the existence of an endogenous autocrine/paracrine chondroprotective mechanism against stimuli inducing chondrocyte apoptosis via the intrinsic/mitochondrial pathway (13). However, it remains unclear whether ER stress is involved in cartilage apoptosis and OA pathogenesis. Therefore, the aim of the present study was to determine whether ER stress is involved in chondrocyte apoptosis and whether it is associated with the degree of cartilage degeneration in OA patients.

Tauroursodeoxycholic acid (TDA) is a hydrophilic bile acid that is normally produced endogenously in humans at extremely low levels. TDA is formed in the conjugation pathway of ursodeoxycholic acid, which is commonly used as a bile acid replacement therapy for the treatment of certain cholestatic syndromes (14). Notably, recent studies have shown 
that TDA can inhibit ER stress in vitro and in vivo (15). In addition, Chen et al (16) demonstrated that TDA regulates ER function and reduces cell apoptosis by decreasing ER stress.

Therefore, we hypothesized that TDA may alleviate OA by protecting chondrocytes and preventing apoptosis through regulation of ER stress. Therefore, chondrocyte function was assessed and the cartilage was observed under ER stress conditions following treatment with tunicamycin. Of note, the effects of TDA on tunicamycin-induced ER stress were evaluated.

In the present study treatment with tunicamycin caused ER stress. Significant changes were observed in cells: High expression of ER stress markers, reduced cell proliferation, increased apoptosis and decreased synthesis of type II collagen. These effects were alleviated by treatment with TDA. These findings provide new insights on the mechanisms underlying ER stress in cartilage degeneration and osteoarthritis development. Additionally, the data provide a basis for new drug development for the prevention and treatment of osteoarthritis.

\section{Materials and methods}

Study subjects. Patients were diagnosed according to the 1995 American Academy of Rheumatology established diagnostic criteria for OA (17). Articular cartilage samples were collected from 18 patients ( 9 males and 9 females) aged between 18 and 65 years, who were diagnosed for OA and underwent total knee arthroplasty in the Department of Orthopaedic Surgery, Peking University First Hospital (Beijing, China) between January and September 2009. All the patients were informed of the purpose of the experiment and provided signed consent. All the procedures were approved by the Institute Review Board and Human Subjects Committee of Peking University First Hospital. The degree of cartilage degeneration was graded using a modified Outerbridge system (18).

Specimen processing. The cartilages obtained from surgery were rinsed with phosphate-buffered saline (PBS) and several blocks of level -I, -II and -III tissue (Outerbridge system) were cut aseptically in a frozen state. Three blocks were obtained for each level with cross-sectional areas of $\sim 60 \mathrm{~mm}^{2} /$ piece, and used for histological examination, tissue protein extraction and RNA extraction. The remaining specimens were stored at $-70^{\circ} \mathrm{C}$.

Histology. Cartilage blocks with subchondral bone were obtained and immersed in decalcification solution composed of neutral formalin and $10 \%$ ethylenediaminetetraacetic acid (EDTA). The medium was changed weekly to soften the subchondral bone. Subsequently, cartilages were embedded, dewaxed and cut into 5- $\mu \mathrm{m}$ sections. Terminal deoxynucleotidyltransferase-mediated dUTP nick end labelling (TUNEL) staining of the samples was carried out using the TUNEL staining kit (Roche Diagnostics, Basel, Switzerland), according to the manufacturer's instructions, to measure cartilage cell apoptosis; apoptotic nuclei appeared brown. Analysis was performed in a double-blinded manner through continuous observation of 5-10 high power fields, where the cells below the tide line were counted. The results are expressed as average number of apoptotic cells/unit.
Cell culture and treatments. The articular cartilages were minced and digested at $37^{\circ} \mathrm{C}$ using $0.2 \% \mathrm{w} / \mathrm{v}$ collagenase II (Santa Cruz Biotechnology, Inc., Dallas, TX, USA) in Dulbecco's modified Eagle's medium (DMEM) supplemented with $10 \%$ fetal bovine serum (FBS). Chondrocytes were collected after 12-16 $\mathrm{h}$ and cultured in DMEM supplemented with $10 \% \mathrm{FBS}, 50 \mathrm{mg} / \mathrm{ml}$ ascorbic acid, $100 \mathrm{U} / \mathrm{ml}$ penicillin and $100 \mathrm{mg} / \mathrm{ml}$ streptomycin at $37^{\circ} \mathrm{C}$ in a $5 \% \mathrm{CO}_{2}$ incubator. When cells were close to $100 \%$ confluency, they were passaged following trypsinization ( $0.05 \%$ trypsin and $0.02 \%$ EDTA). Subsequently, the cells were harvested following the second passage and cultured in 6-well plates at a density of $0.6 \times 10^{6}$ cells/well. The chondrocytes were divided into three groups: CON group, no treatment; ERS group treatment with $2 \mu \mathrm{mol} / \mathrm{l}$ tunicamycin on day 4 after seeding; and TDA group, treatment with $200 \mu \mathrm{mol} / \mathrm{l}$ TDA $4 \mathrm{~h}$ after tunicamycin treatment.

Cell proliferation assay. A CCK-8 Cell Proliferation assay kit (Promega, Beijing, China) was used to determine viability of the cells, according to the manufacturer's instructions. Briefly, $1 \times 10^{4}$ cells/well were seeded in 6 -well plates and $20 \mu 1$ of CCK-8 solution was added into each well at each time-point (days 1-9) followed by incubation at $37^{\circ} \mathrm{C}$ for $4 \mathrm{~h}$. Finally, cell viability was measured using a microplate reader (\#M581789; Westingarea, Shanghai, China).

Apoptosis assay. Apoptotic cells were quantified by Annexin V-FITC and propidium iodide (PI) staining. Detection of early apoptosis in chondrocytes was performed using a kit from Nanjing KeyGen Biotech. Co., Ltd. (Nanjing, China) according to the manufacturer's instructions. In brief, chondrocytes $\left(10^{5}\right.$ cells $\left./ \mathrm{ml}\right)$ were harvested and centrifuged at $450 \mathrm{x} \mathrm{g}$ for $5 \mathrm{~min}$ at $4^{\circ} \mathrm{C}$. The cells were washed twice with PBS and the pellets were resuspended in $100 \mu \mathrm{l}$ ice-cold binding buffer. Subsequently, cells were incubated with $5 \mu 1$ Annexin $\mathrm{V}$ and $5 \mu \mathrm{l} \mathrm{PI}$ for $10 \mathrm{~min}$ at room temperature in the dark. Following washing, cells were resuspended in $400 \mu \mathrm{l}$ binding buffer and analyzed on a FACScan flow cytometer (BD Biosciences, San Jose, CA, USA). The negative control was set for each sample, with the cells incubated with binding buffer alone.

TUNEL staining. The apoptosis index of the chondrocytes was assessed using a TUNEL staining kit (Maxim, Fujian, China) according to the manufacturer's instructions. The samples were examined under microscopy (Olympus CX31-12C04; Olympus, Tokyo, Japan) and stained cells were counted in 6 random visual fields.

Western blot analysis. Cells collected by centrifugation and the cartilage, which was ground to powder in liquid nitrogen, were resuspended in radioimmunoprecipitation assay buffer in the presence of the protease inhibitors. Following lysate clarification, protein concentration was determined using the bicinchoninic acid assay reagent kit (Pierce, Rockford, IL, USA) with bovine serum albumin as the standard. Subsequently, $30 \mu \mathrm{g}$ of total protein was denatured at $95^{\circ} \mathrm{C}$ for $5 \mathrm{~min}$ in $2 \%$ SDS sample buffer in the presence of NuPage reducing agent (Invitrogen, Carlsbad, CA, USA). Equivalent 


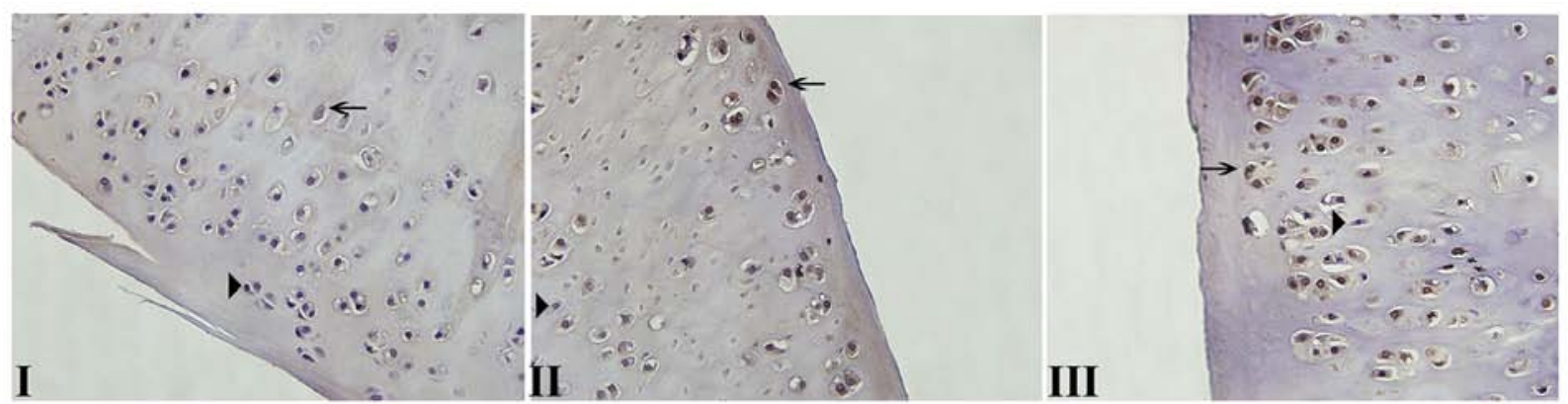

Figure 1. Cell apoptosis in the cartilages of patients with different osteoarthritis (OA) grades was examined by terminal deoxynucleotidyltransferase-mediated dUTP nick end labelling (TUNEL) staining. Filled triangles show the normal cells stained blue by hematoxylin, and arrows indicate the apoptotic cells stained yellow by TUNEL (magnification, x100). Normal cell nuclei were stained in blue by hematoxylin and showed a good shape. However, apoptotic chondrocytes showed an uneven distribution in the articular cartilage, and mainly concentrated in the surface and shallow regions of the cartilage. Apoptotic nucleus showed condensation, loss of the oval shape and an uneven chromatin distribution. I, II and III indicate the different disease stages.

amounts of protein were resolved on $12 \%$ SDS-polyacrylamide gels and electroblotted onto polyvinylidene difluoride (Pierce) using the Invitrogen $\mathrm{X}$ cell electrophoresis and blotting system (Invitrogen). Following blocking with 5\% (w/v) non-fat milk in wash buffer (Tris-buffered saline containing $0.1 \%$ Tween-20) for $2 \mathrm{~h}$, membranes were incubated with rabbit monoclonal anti-glucose regulated protein 78 (GRP78) (sc-13968; 1:500), rabbit monoclonal anti-caspase-12 $(1: 1,000)$, goat monoclonal anti-growth arrest and DNA-damage-inducible gene 153 (GADD153) (sc-575; 1:500), rabbit anti-collagen II (sc-7763; 1:1,000), and rabbit monoclonal anti-actin (sc-7210; 1:2,000) (all from Santa Cruz Biotechnology, Inc.). Following four washes, the membranes were incubated with horseradish peroxidase-conjugated anti-rabbit or anti-goat secondary antibodies at 1:5,000 dilution and immunoreactivity was visualized using an ECL system (Pierce).

Reverse transcription-quantitative polymerase chain reaction (RT- $q P C R)$. Total RNA was prepared from chondrocytes cultured in vitro or harvested from the cartilage using the TRIzol Reagent (Invitrogen) according to the manufacturer's instructions. To amplify 133-base pair (bp) GRP787, 182-bp GADD153, 244-bp caspase-12, 286-bp collagen II and 200-bp glyceraldehyde 3-phosphate dehydrogenase (GAPDH) cDNA fragments, PCR primers were synthesized by Genosys (The Woodlands, TX, USA): GRP78 sense, 5'-TCC TAT GTC GCC TTC ACT-3' and antisense, 5'-ACA GAC GGG TCA TTC CAC-3'; GADD153 sense, 5'-CTG ACC AGG GAA GTA GAG G-3' and antisense, 5'-TGC GTA TGT GGG ATT GAG-3'; caspase-12 sense, 5'-AAT CTG TGG GAC CAA GCA-3' and antisense, 5'-GAG CCT TTG TAA CAG CAT CA-3'); GAPDH sense, 5'-ACC CAG AAG ACT GTG GAC TT-3' and antisense, 5'-TTC TAG ACG GCA GGT CAG GT-3'; and collagen II sense, 5'-CCA CAC TCA ATC CCT CAA C-3' and antisense, 5'-GCT GCT CCA CCA GTT CTT C-3'. The samples were first denatured at $95^{\circ} \mathrm{C}$ for $30 \mathrm{sec}$, followed by 32 cycles of $95^{\circ} \mathrm{C}$ for $30 \mathrm{sec}, 60^{\circ} \mathrm{C}$ for $30 \mathrm{sec}$ and $72^{\circ} \mathrm{C}$ for $30 \mathrm{sec}$. The last cycle was followed by an additional incubation for $7 \mathrm{~min}$ at $72^{\circ} \mathrm{C}$. Analysis of amplicons was accomplished on $1 \%$ agarose gel containing $0.2 \mu \mathrm{g} / \mu \mathrm{l}$ ethidium bromide and amplicons were visualized under a UV trans-luminator. The densitometric analysis of the PCR products was performed by Bio-Rad Quantity One on GS-800 Imaging Densitometer (both from Bio-Rad, Hercules, CA, USA) and all the values were standardized against the GAPDH product. The cycle threshold (CT) was defined as the cycle number at which the fluorescence generated by cleavage of the probe exceeded a fixed threshold above the baseline. Final values, which were expressed as fold difference relative to the GAPDH gene $(\mathrm{N})$, were calculated with the following formula: $\mathrm{N}=2^{\text {(C-T)gene }} / 2^{\text {(C-T)GAPDH }}$, where $\mathrm{C}$ is the $\mathrm{CT}$ for GRP78, GAPDH in control samples; and $\mathrm{T}$ is the CT for GRP78, GAPDH in treatment samples. All the PCR reactions were performed using the LightCycler FastStart DNA Master SYBR-Green I kit and the Cepheid SmartCycler real-time PCR cycler (Cepheid Inc., Sunnyvale, CA, USA). The cycling conditions were as follows: Initial denaturation at $95^{\circ} \mathrm{C}$ for $10 \mathrm{~min} ; 40$ cycles at $95^{\circ} \mathrm{C}$ for $15 \mathrm{sec}, 60^{\circ} \mathrm{C}$ for $5 \mathrm{sec}$ and $72^{\circ} \mathrm{C}$ for $10 \mathrm{sec}$. Experiments were performed in triplicate for each data point, and for all the experiments the controls were included without templates.

Statistical analysis. The data are presented as mean \pm standard error for the number of experiments indicated. One-way analysis of variance was used to determine significant differences among groups. All the statistical analyses were generated using the SPSS software 11.0 (SPSS Inc., Chicago, IL, USA) and figures were plotted using the Origin 7.5 software (OriginLab Corp., Northampton, MA, USA). P $<0.05$ was considered to indicate a statistically significant difference.

\section{Results}

Apoptosis of chondrocytes in the cartilage of patients with $O A$ associated with the grade of $O A$. Normal cell nuclei stained in blue by hematoxylin maintained a good shape (Fig. 1). Apoptotic chondrocytes showed an uneven distribution in articular cartilage and were mainly concentrated on the surface and shallow regions. Apoptotic cell nuclei exhibited condensation, loss of the oval shape and uneven chromatin distribution. Notably, the number of apoptotic chondrocytes was increased with the aggravation of cartilage degeneration: The apoptosis rate of chondrocytes in cartilage specimens was $8.2,14.1$ and $23.7 \%$ in grades I, II and III, respectively.

Type II collagen (Col II) expression and ER stress in cartilage chondrocytes of patients with $O A$ is associated with the $O A$ 

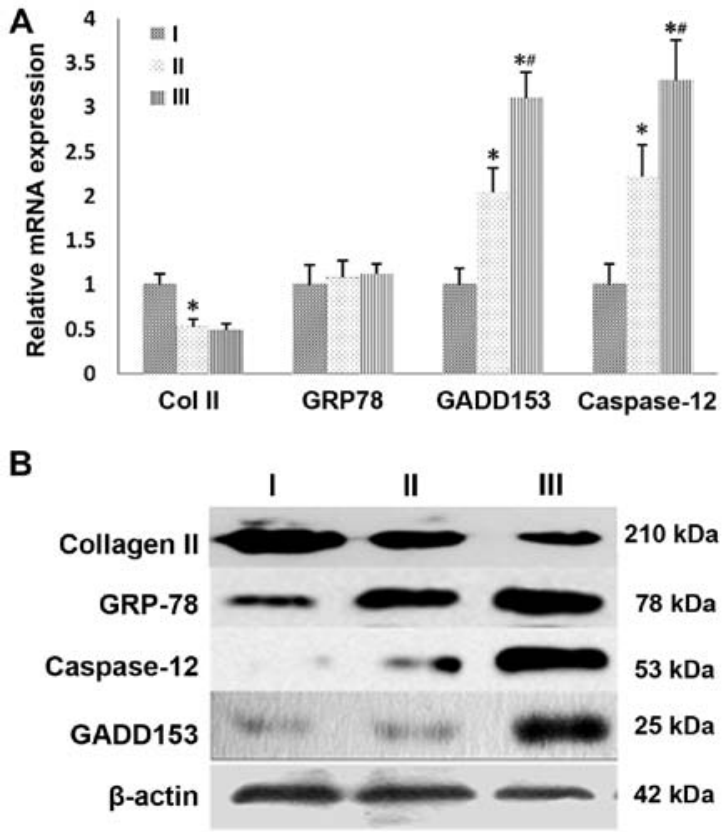

Figure 2. (A) mRNA and (B) protein expression of type II collagen (Col II), glucose-regulated protein $78(G R P 78)$, growth arrest and DNA-damageinducible gene 153 (GADD153) and caspase-12 in cartilages from patients with different osteoarthritis grades. ${ }^{*} \mathrm{P}<0.05$ vs. I; and ${ }^{\#} \mathrm{P}<0.05$ vs. II.

grade. The mRNA levels of Col II were decreased by 47 and $51 \%$ in grades II $(\mathrm{P}<0.01)$ and III $(\mathrm{P}<0.01)$, respectively, as compared to grade I. However, no significant difference was observed between grades II and III, indicating the reduced ability of chondrocytes to synthesize the extracellular matrix. The GRP78 gene expression was increased by 8 and $12 \%$ in grades II $(\mathrm{P}<0.01)$ and III $(\mathrm{P}<0.01)$, respectively, as compared to grade I. In the case of GADD153, gene expression was increased by 104 and $210 \%$ in grades II $(\mathrm{P}<0.01)$ and III $(\mathrm{P}<0.01)$, respectively, as compared to grade I. Finally, caspase-12 gene expression was increased by 121 and $230 \%$ in grades II $(\mathrm{P}<0.01)$ and III $(\mathrm{P}<0.01)$, respectively, as compared to grade I (Fig. 2A).

As for gene expression, Col II protein levels were decreased by 23 and $31 \%$ in grades II $(\mathrm{P}<0.01)$ and III $(\mathrm{P}<0.01)$, respectively, as compared to grade I, and no significant difference was obtained between grades II and III. The protein expression of GRP78 was increased by 23 and $27 \%$ in grades II $(\mathrm{P}<0.01)$ and III cartilage tissue $(\mathrm{P}<0.01)$, respectively, as compared with grade I. As for caspase-12, protein levels were increased by 143 and $210 \%$ in grades II $(\mathrm{P}<0.01)$ and III $(\mathrm{P}<0.01)$, respectively, as compared to grade I. In the case of GADD153, protein expression was increased by 98 and $180 \%$ in grades II $(\mathrm{P}<0.01)$ and III ( $\mathrm{P}<0.01)$, respectively, as compared to grade I (Fig. 2B).

TDA treatment results in cell proliferation recovery and a decrease in apoptosis. Cells that were adherent after $24 \mathrm{~h}$ of culture began to form spindles and gradually became polygonal, with large round nuclei and abundant cytoplasm containing secretory granules. Cell processes began to resume over one week and cells were surrounded by matrix-like material deposition. Medium floating apoptotic cells were infrequently observed. Considering that tunicamycin stimulated the growth of chondrocytes with a poor morphology, more cells were collapsed with medium large apoptotic cells floating. TDA-treated chondrocytes grew well and became more angular and adherent with rich cytoplasm (Fig. 3A), The number of medium floating apoptotic cells was significantly reduced compared with the tunicamycin-treated group. Tunicamycin induced ER stress in chondrocytes (Fig. 3B), which resulted in a marked reduction in cell proliferation $(\mathrm{P}<0.01)$. However, cell proliferation was significantly higher in tunicamycin-treated cells following TDA treatment.

Following chondrocyte treatment, cells were stained with Annexin V/PI and analyzed by flow cytometry. Apoptosis rates were 9.2 and $46.7 \%$ in the CON and ERS groups, respectively. However, the apoptosis rate decreased to $28.3 \%$ in the TDA group (Fig. 3C).

Effect of tunicamycin and TDA treatment on type II collagen expression in chondrocytes. Type II collagen mRNA levels were decreased by $82 \%(\mathrm{P}<0.01)$ in the ERS group compared to the CON group, and increased by $170 \%(\mathrm{P}<0.01)$ in the TDA group as compared to the ERS group (Fig. 4A).

Similarly, type II collagen protein expression was decreased by $84 \%(\mathrm{P}<0.01)$ in the ERS group as compared to the CON group. However, type II collagen protein levels were increased by $245 \%(\mathrm{P}<0.01)$ in the TDA group as compared to the ERS group (Fig. 4B).

TDA inhibits ER stress induced by tunicamycin in chondrocytes. The gene expression levels of GRP78, GADD153 and caspase-12 were increased by 108,112 and $113 \%(\mathrm{P}<0.01)$, respectively, in chondrocytes treated with tunicamycin compared with the CON group (Fig. 5A). Of note, GRP78, GADD153 and caspase-12 mRNA levels were decreased by 40,44 and $34 \%(\mathrm{P}<0.01)$, respectively, in the TDA group as compared to chondrocytes treated with tunicamycin only.

Similarly, GRP78, GADD153 and caspase-12 protein expression was increased by 52,71 and $54 \%(\mathrm{P}<0.01)$, respectively, in chondrocytes treated with tunicamycin associated with the CON group. Notably, GRP78, GADD153 and caspase-12 protein levels were decreased by $23.7,36.3$ and $30.1 \%$ ( $\mathrm{P}<0.01)$, respectively, in the TDA group as compared to the ERS group (Fig. 5B).

\section{Discussion}

In the present study, the presence of ER stress was demonstrated in the cartilage of patients with osteoarthritis and a positive correlation was confirmed between ER stress and osteoarthritis severity. Osteoarthritis was mainly graded using the Outerbridge system and was manifested by mRNA and protein expression of ER stress markers, such as GRP78, GADD153 and caspase-12, indicating the involvement of ER stress in the osteoarthritis pathological process.

The ER regulates protein synthesis, folding and transport, and is involved in intracellular calcium homeostasis and a variety of cell signaling pathways (19-21). Thus, changes in intracellular calcium homeostasis results in accumulation of unfolded or misfolded proteins in the ER, leading to ER stress $(7,8)$. GRP78 binds to unfolded proteins that aggregate in the ER and activates downstream effectors, which suppress 

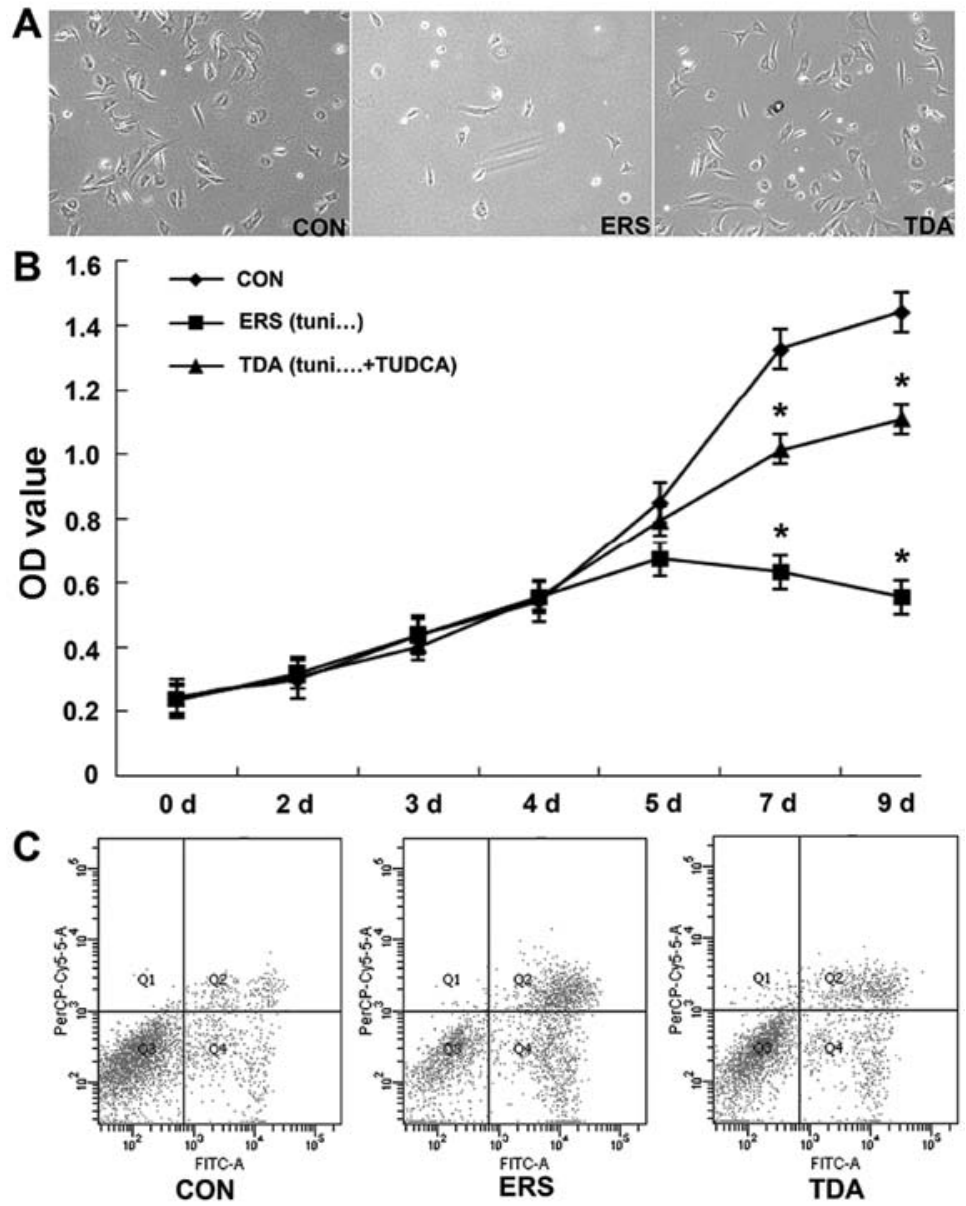

Figure 3. Cell growth, proliferation and apoptosis. (A) Cells of the control (CON), endoplasmic reticulum stress (ERS) and tunicamycin (TDA) groups were imaged at day (d) 7 under a microscope (magnification, x100); (B) cell proliferation was measured by the CCK-8 assay; (C) chondrocytes were stained with Annexin V/propidium iodide and analyzed by flow cytometry. ${ }^{*} \mathrm{P}<0.05$ vs. corresponding CON group.
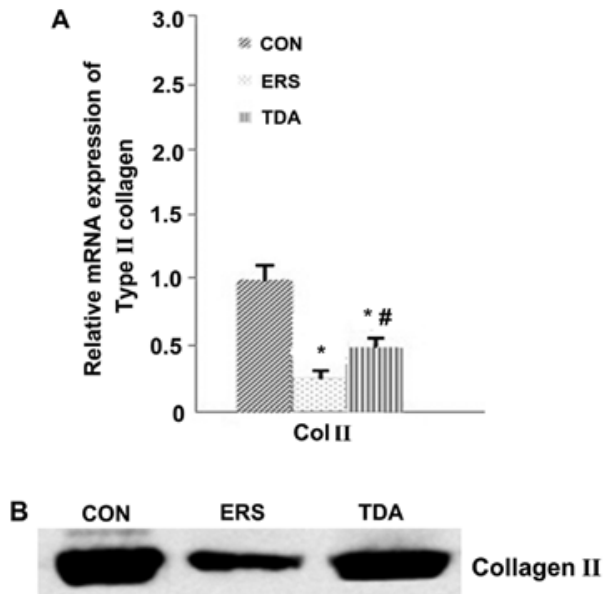

Figure 4. Type II collagen expression in chondrocytes. (A) mRNA and (B) protein levels were determined in the cells collected from the control $(\mathrm{CON})$, endoplasmic reticulum stress (ERS) and tunicamycin (TDA) groups at day $7 .{ }^{*} \mathrm{P}<0.05$ vs. $\mathrm{CON}$ group; and ${ }^{\prime \prime} \mathrm{P}<0.05$ vs. ERS group.

protein synthesis and express stress response proteins to restore the ER protein processing capacity, the redox balance and calcium homeostasis $(19,22,23)$. Of note, as cartilage degeneration worsened, GRP78 expression increased gradually, as shown, indicating that larger quantities of unfolded proteins

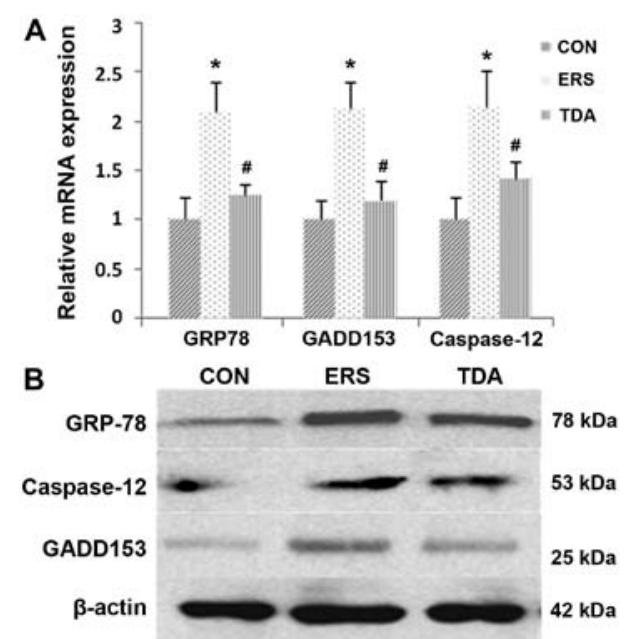

Figure 5. Glucose-regulated protein 78 (GRP78), growth arrest and DNA-damage-inducible gene 153 (GADD153) and caspase-12 expression in chondrocytes. (A) mRNA and (B) protein levels were determined in cells collected from the control (CON), endoplasmic reticulum stress (ERS) and tunicamycin (TDA) groups at day 7 . ${ }^{*} \mathrm{P}<0.05$ vs. $\mathrm{CON}$ group; and ${ }^{\#} \mathrm{P}<0.05$ vs. ERS group.

could be bound in order to protect chondrocytes during the stress. However, due to the persistence of external stimulation, ER stress caused apoptosis. The present study found that ER 
apoptosis markers were highly expressed in level III cartilage, including GADD153 and activated caspase-12. These observations indicated that during ER stress, the chondrocytes decreased the synthesis of extracellular matrix, decreasing the cartilage thickness and increasing the fibrous tissue. With the ER stress pathway activation, chondrocyte apoptosis gradually increased, resulting in decreased cell number and the observed irregularly arranged cartilage.

With the Outerbridge grade increase, it was also demonstrated that osteoarthritis became more serious, with TUNEL-positive cells appearing more frequently, an indication of increased apoptosis. In addition, reduced mRNA and protein expression of type II collagen and the main matrix proteins was shown in osteoarthritis-affected cartilages. These findings indicated a significantly reduced condrocyte activity and increased apoptosis during osteoarthritis development. The association between condrocyte apoptosis and ER stress is well documented $(10,24,25)$. Notably, protein folding inhibition in the ER lumen by tunicamycin resulted in an ER stress response as evidenced by increased GADD153 protein and gene expression, and PERK and eIF2- $\alpha$ phosphorylation, which resulted in apoptosis (26). Therefore, the present results suggest that ER stress could lead to cartilage cell apoptosis by affecting chondrocyte activity along with a gradual degeneration of cartilage and osteoarthritis development.

As shown, treatment of chondrocytes with tunicamycin resulted in ER stress, evidenced by the increased expression of GRP78 at the gene and protein levels. As a result, cell function was damaged, leading to reduced type II collagen synthesis and increased expression of caspase-12 and GADD153. Flow cytometry data confirmed the increased apoptosis following ER stress induction by tunicamycin. Of note, administration of TDA to chondrocytes pretreated with tunicamycin caused a relative decline in GRP78 expression, i.e. decreased ER stress concomitantly with increased type II collagen levels and decreased expression of apoptosis markers such as caspase-12 and GADD153. Furthermore, flow cytometry data showed that the tunicamycin-induced apoptosis was significantly decreased following treatment with TDA. Therefore, we believe that TDA reduces ER stress in chondrocytes, protects ER function in protein synthesis and folding, and reduces apoptosis.

Multiple studies have demonstrated the modulatory role of TDA on the ER function, in vitro and in vivo. TDA was shown to attenuate tunicamycin-induced ER stress, autophagy and cell death in cultured hepatocytes $(27,28)$. In addition, TDA protects rat pancreatic $\beta$-cells (INS- 1 cells) from palmitate-induced injury, which may be due to the amelioration of ER stress among others (29), demonstrating a potential application in the treatment of type 2 diabetes (15). Chen et al (16) proposed that ER stress has an important role in AGEs-induced apoptosis, which was prevented by TDA following blocking of an ER stress-mediated apoptotic pathway in chondrocytes. Taken together, these findings demonstrate the important role of ER stress in chondrocyte apoptosis.

In conclusion, ER stress and chondrocyte apoptosis are involved in the development of osteoarthritis. However, there is a possibility of chondrocyte apoptosis induction by mechanisms other than ER stress. In addition, whether the two other apoptotic pathways were induced was not investigated. The ER markers GADD153, caspase-12 and GRP78, which are independent and specific for ER stress, were detected. Therefore, the study partly provides a mechanism underlying ER stress in cartilage degeneration and osteoarthritis development.

\section{Acknowledgements}

The present study was supported by the National Natural Science Fundation of China (grant no. 30772198).

\section{References}

1. Loeser RF: Aging and osteoarthritis: The role of chondrocyte senescence and aging changes in the cartilage matrix. Osteoarthritis Cartilage 17: 971-979, 2009.

2. Creamer P and Hochberg MC: Osteoarthritis. Lancet 350: 503-508, 1997.

3. Blanco FJ, Guitian R, Vázquez-Martul E, de Toro FJ and Galdo : Osteoarthritis chondrocytes die by apoptosis. A possible pathway for osteoarthritis pathology. Arthritis Rheum 41: 284-289, 1998.

4. Hashimoto S, Ochs RL, Komiya S and Lotz M: Linkage of chondrocyte apoptosis and cartilage degradation in human osteoarthritis. Arthritis Rheum 41: 1632-1638, 1998.

5. Kühn K, D'Lima DD, Hashimoto S and Lotz M: Cell death in cartilage. Osteoarthritis Cartilage 12: 1-16, 2004.

6. Aigner T, Kurz B, Fukui N and Sandell L: Roles of chondrocytes in the pathogenesis of osteoarthritis. Curr Opin Rheumatol 14: 578-584, 2002.

7. Boyce $\mathrm{M}$ and Yuan J: Cellular response to endoplasmic reticulum stress: A matter of life or death. Cell Death Differ 13: 363-373, 2006.

8. Brostrom MA and Brostrom CO: Calcium dynamics and endoplasmic reticular function in the regulation of protein synthesis: Implications for cell growth and adaptability. Cell Calcium 34: 345-363, 2003.

9. Archer CW and Francis-West P: The chondrocyte. Int J Biochem Cell Biol 35: 401-404, 2003.

10. Yang L, Carlson SG, McBurney D and Horton WE Jr: Multiple signals induce endoplasmic reticulum stress in both primary and immortalized chondrocytes resulting in loss of differentiation, impaired cell growth, and apoptosis. J Biol Chem 280: 31156-31165, 2005.

11. Ruiz-Romero C, Carreira V, Rego I, Remeseiro S, López-Armada MJ and Blanco FJ: Proteomic analysis of human osteoarthritic chondrocytes reveals protein changes in stress and glycolysis. Proteomics 8: 495-507, 2008.

12. Nugent AE, Speicher DM, Gradisar I, McBurney DL, Baraga A, Doane KJ and Horton WE Jr: Advanced osteoarthritis in humans is associated with altered collagen VI expression and upregulation of ER-stress markers Grp78 and bag-1. J Histochem Cytochem 57: 923-931, 2009.

13. Intekhab-Alam NY, White OB, Getting SJ, Petsa A, Knight RA, Chowdrey HS, Townsend PA, Lawrence KM and Locke IC: Urocortin protects chondrocytes from NO-induced apoptosis: A future therapy for osteoarthritis? Cell Death Dis 4: e717, 2013.

14. Hardison WG and Grundy SM: Effect of ursodeoxycholate and its taurine conjugate on bile acid synthesis and cholesterol absorption. Gastroenterology 87: 130-135, 1984.

15. Ozcan U, Yilmaz E, Ozcan L, Furuhashi M, Vaillancourt E, Smith RO, Görgün CZ and Hotamisligil GS: Chemical chaperones reduce ER stress and restore glucose homeostasis in a mouse model of type 2 diabetes. Science 313: 1137-1140, 2006.

16. Chen Y, Liu CP, Xu KF, Mao XD, Lu YB, Fang L, Yang JW and Liu C: Effect of taurine-conjugated ursodeoxycholic acid on endoplasmic reticulum stress and apoptosis induced by advanced glycation end products in cultured mouse podocytes. Am J Nephrol 28: 1014-1022, 2008.

17. Hochberg MC, Altman RD, Brandt KD, Clark BM, Dieppe PA, Griffin MR, Moskowitz RW and Schnitzer TJ; American College of Rheumatology: Guidelines for the medical management of osteoarthritis. Part II. Osteoarthritis of the knee. Arthritis Rheum 38: 1541-1546, 1995.

18. Outerbridge RE: The etiology of chondromalacia patellae. J Bone Joint Surg Br 43-B: 752-757, 1961.

19. Xu C, Bailly-Maitre B and Reed JC: Endoplasmic reticulum stress: Cell life and death decisions. J Clin Invest 115: 2656-2664, 2005. 
20. Rao RV, Ellerby HM and Bredesen DE: Coupling endoplasmic reticulum stress to the cell death program. Cell Death Differ 11: 372-380, 2004

21. Iwawaki T, Akai R, Kohno K and Miura M: A transgenic mouse model for monitoring endoplasmic reticulum stress. Nat Med 10: 98-102, 2004.

22. Oyadomari S and Mori M: Roles of CHOP/GADD153 in endoplasmic reticulum stress. Cell Death Differ 11: 381-389, 2004.

23. Wang XZ, Lawson B, Brewer JW, Zinszner H, Sanjay A, Mi LJ, Boorstein R, Kreibich G, Hendershot LM and Ron D: Signals from the stressed endoplasmic reticulum induce C/EBP-homologous protein (CHOP/GADD153). Mol Cell Biol 16: 4273-4280, 1996.

24. Oliver BL, Cronin CG, Zhang-Benoit Y, Goldring MB and Tanzer ML: Divergent stress responses to IL-1beta, nitric oxide, and tunicamycin by chondrocytes. J Cell Physiol 204: 45-50, 2005.

25. Matsuo M, Nishida K, Yoshida A, Murakami T and Inoue $\mathrm{H}$ : Expression of caspase- 3 and -9 relevant to cartilage destruction and chondrocyte apoptosis in human osteoarthritic cartilage. Acta Med Okayama 55: 333-340, 2001.
26. Pelletier JP, Jovanovic DV, Lascau-Coman V, Fernandes JC Manning PT, Connor JR, Currie MG and Martel-Pelletier J: Selective inhibition of inducible nitric oxide synthase reduces progression of experimental osteoarthritis in vivo: Possible link with the reduction in chondrocyte apoptosis and caspase 3 level. Arthritis Rheum 43: 1290-1299, 2000.

27. Xie Q, Khaoustov VI, Chung CC, Sohn J, Krishnan B, Lewis DE and Yoffe B: Effect of tauroursodeoxycholic acid on endoplasmic reticulum stress-induced caspase-12 activation. Hepatology 36: 592-601, 2002.

28. Zhang J, Morris MW Jr, Dorsett-Martin WA, Drake LC and Anderson CD: Autophagy is involved in endoplasmic reticulum stress-induced cell death of rat hepatocytes. J Surg Res 183: 929-935, 2013.

29. Zhu Q, Zhong JJ, Jin JF, Yin XM and Miao H: Tauroursodeoxycholate, a chemical chaperone, prevents palmitate-induced apoptosis in pancreatic $\beta$-cells by reducing ER stress. Exp Clin Endocrinol Diabetes 121: 43-47, 2013. 